\title{
Interferon- $\gamma$-Inducible Protein 10 for Diagnosis of Tuberculosis in Children
}

\author{
Stefani Candra Firmanti ${ }^{1,2}$, Rina Triasih ${ }^{3}$, Tri Wibawa ${ }^{4}$, Sofia Mubarika Haryana ${ }^{5, *}$ \\ ${ }^{1}$ Doctoral Program, Faculty of Medicine, Public Health and Nursing, Universitas Gadjah Mada, Jl. Farmako, Sekip Utara, Yogyakarta 55281, Indonesia \\ ${ }^{2}$ Department of Microbiology, Faculty of Medicine, Universitas Diponegoro, Jl. Prof. Soedarto, Semarang 50275, Indonesia \\ ${ }^{3}$ Department of Paediatrics, Faculty of Medicine, Public Health and Nursing, Universitas Gadjah Mada/Dr. Sardjito Hospital, J1. Farmako, Sekip Utara, \\ Yogyakarta 55281, Indonesia \\ ${ }^{4}$ Department of Microbiology, Faculty of Medicine, Public Health and Nursing, Universitas Gadjah Mada, Jl. Farmako, Sekip Utara, \\ Yogyakarta 55281, Indonesia \\ ${ }^{5}$ Department of Histology and Cell Biology, Faculty of Medicine, Public Health and Nursing, Universitas Gadjah Mada, Jl. Farmako, Sekip Utara, \\ Yogyakarta 55281, Indonesia \\ *Corresponding author. E-mail: sofia.mubarika@gmail.com
}

Received date: Aug 28, 2019; Revised date: Oct 21, 2019; Accepted date: Oct 28, 2019

\section{Abstract}

B ACKGROUND: The diagnosis of tuberculosis (TB) in children is challenging by the absence of a practical gold standard. Interferon (IFN)- $\gamma$ inducible protein 10 (IP-10) is a chemokine that may serve as the leading candidate marker in child TB diagnosis. The aim of this study is to assess the diagnostic value of IP-10 in the diagnosis of TB in children.

METHODS: We recruited eligible symptomatic and asymptomatic children aged $<15$ years actively by contact investigation and passively from inpatient and outpatient clinics in two hospitals, in Yogyakarta, Indonesia. We conducted clinical examination and chest $\mathrm{X}$-ray in all eligible children. Sputum smear and the rapid molecular TB test were performed in children with TB symptoms. All participants underwent blood sampling for IFN- $\gamma$ Release Assay and IP-10 test.
RESULTS: A total of 79 children were recruited to this study. Twelve children were with TB disease, 16 with latent TB infection (LTBI), 40 were TB-exposed only and 11 were non-TB. Children with evidence of TB infection either with TB disease or LTBI had higher levels of antigen-stimulated IP-10 compared to non-infected children, both TB exposed only and non-TB ( $p=0.000)$. A cut-off $408.74 \mathrm{pg} / \mathrm{mL}$ for antigen-stimulated IP-10 showed high diagnostic accuracy for diagnosis of TB infection (AUC: 0.97, 95\% CI: 0.921.00, sensitivity: $92.3 \%$, and specificity: $91.9 \%$ ). However, the stimulated levels of IP-10 between children with TB disease and LTBI were not significantly different $(p=0.268)$.

CONCLUSION: IP-10 performed well to diagnose TB infection in children. However, it cannot be used to differentiate TB infection from TB disease.

KEYWORDS: IFN- $\gamma$, IP-10, latent TB, active TB, children

Indones Biomed J. 2020; 12(1): 19-26

\section{Introduction}

Tuberculosis (TB) contributes significantly to morbidity and mortality in children, especially in TB endemic settings. (1) Globally, incidence of TB in children each year has reached nearly 1 million and children (0-14 years old) represent about $10-11 \%$ of all TB cases. The World Health Organization (WHO) estimated that 230,000 children died of TB in 2017, including 52,000 children with human immunodeficiency virus (HIV) associated TB.(2) However, the actual burden of TB in children is likely higher given the challenge in the diagnosis of TB in children.

TB diagnosis in children remains a significant challenge due to the lack of an established practical gold standard. Even with advanced molecular technologies, microbiological confirmation is often difficult because of the paucibacillary nature of the disease. Immunodiagnostic tests 
may improve performance of diagnosis, but the current tests cannot discriminate infection from disease.(3) Accurate, timely, simple, and cheap diagnostic tools to distinguish patients with active disease from latent infection is an important priority for TB treatment in children. Adequate identification of active and latent TB cases is essential to direct effective treatment to cure and prevent relapse in patients with active disease or to provide appropriate preventive therapy.(4)

Interferon (IFN)- $\gamma$ release assays (IGRA) are expected to improve the specificity of tuberculin skin testing (TST) for detection of latent TB infection. However, IGRA are costly and technically complex. The use of IGRA has not been recommended globally, limited by a countries' income status since IGRA have suboptimal sensitivity when used in low and middle income countries, where the burden of TB is highest. Accuracy of IGRA is also compromised in the patients who are young and immunosuppressed.(5)

The IFN- $\gamma$-inducible protein 10 (IP-10) is one of the promising candidate biomarkers which can improve the IGRA sensitivity and does not reduce specificity.(6) IP-10 acts as downstream marker of IFN- $\gamma$, which is a commonly used marker for cell-mediated immunity. IP-10 promotes Th1 cells migration to infection sites by a process of binding with C-X-C motif chemokine receptor 3 (CXCR3) on $\mathrm{T}$ cells. Compared to IFN- $\gamma$, IP-10 is expressed in larger amounts while retaining the signal-to-noise ratio, and the protein's stability in dried blood spots allows for innovation with simplified test platforms, especially lateral flow which implies a potential for a point of care diagnostic test.(7-9) Many clinical reports found that IP-10 release assays are comparable to IGRAs in terms of diagnostic accuracy. (4,9-19) Presently, studies in children are limited and have inconsistent results. In view of these findings, this study aim to assess IP-10 as a novel TB diagnostic biomarker in children in endemic settings, specifically, for differentiating TB disease from TB infection.

\section{Methods}

\section{Study Site and Population}

An observational cross-sectional study was conducted in two hospitals in Yogyakarta, Indonesia from August 2017 to April 2018. We recruited children aged $<15$ years in two approaches: a) passively, from symptomatic children who came to the study sites (inpatient and outpatient) during the study period; and b) actively by inviting children, both symptomatic and asymptomatic, who were in close contact with an adult TB case, who were treated in the study sites. Children who have been treated for TB were excluded. Informed consent was obtained from the parents or guardian before participation in the study.

\section{Symptom Screening and Investigations}

All eligible children, irrespective of symptoms, underwent clinical evaluation including nutritional assessment, chest $\mathrm{X}$-Ray (CXR), and blood sampling for quantiferon-TB gold in-tube (QFT-IT) assay and IP-10 test. Microbiological confirmation of sputum sample was performed in children with symptoms. A study doctor identified the symptom suggestive of TB (persistent cough $>2$ weeks, persistent fever $>2$ weeks, weight loss or failure to thrive recorded in last 3 months, and unexplained lethargy) as a "well-defined" symptom if meeting the criteria as previously described.(20) The nutritional status was assessed based on weight for height (WFH) Z-scores (0-4 years) and percentage of expected WFH ( $\geq 5$ years) from WHO criteria. WFH Z-scores $>-2$ or expected WFH $>90 \%$ indicates well-nourished. Z-scores between -3 and -2 or expected WFH between $70-90 \%$ for moderate wasting and Z-scores $<-3$ or expected percentage WFH $<70 \%$ for severe wasting.(21)

The results of CXR in anteroposterior and lateral views were interpreted by an experienced radiologist and a pediatrician who were blinded to the clinical information. CXR was classified as "consistent with TB" if there was a concordant interpretation of any abnormality radiographic features of $\mathrm{TB}$ by reviewers. For microbiological confirmation, two separate induced sputum sample were collected from all symptomatic children. All sputum specimens were examined for acid-fast bacilli (AFB) and sputum pellets were analyzed for Xpert MTB/RIF assay (Cepheid, Sunnyvale, California).

\section{The QFT-IT Assay}

The QFT-IT examination was done following standard protocol (Qiagen, Hilden, Germany), in which one $\mathrm{mL}$ of blood was drawn into each of three separate QFT-IT tubes: unstimulated tube (Nil), TB Antigen tube (Ag), and phytohemagglutinin tube as mitogen (Mit) and incubated at $37^{\circ} \mathrm{C}$ for 16 to 24 hours. After incubation, plasma supernatants were immediately collected by centrifugation and stored at $-20^{\circ} \mathrm{C}$ until use. IFN- $\gamma$ level was measured by QFT enzyme-linked immunosorbent assay (ELISA). QFT results were interpreted as positive, negative, or indeterminate according to the manufacturer's recommendation. The antigen-dependent and mitogenstimulated IFN- $\gamma$ levels were evaluated by subtracting the 
level of IFN- $\gamma$ in Nil vacutainer from the Ag vacutainer and Mit vacutainer, respectively. QFT results were defined by the antigen-dependent (Ag-Nil) IFN- $\gamma$ value: positive result $(\geq 0.35 \mathrm{IU} / \mathrm{mL})$ and negative result $(<0.35 \mathrm{IU} / \mathrm{mL})$. The results were considered indeterminate if the mitogenstimulated (Mit-Nil) IFN- $\gamma$ value $\leq 0.5 \mathrm{IU} / \mathrm{mL}$ and/or the value of the Nil IFN- $\gamma \geq 8.0 \mathrm{IU} / \mathrm{mL}$. For comparison with IP-10 levels, the IFN- $\gamma$ results were reported in $\mathrm{pg} / \mathrm{mL} ; 1$ $\mathrm{IU} / \mathrm{mL}$ of IFN- $\gamma$ equals to $50 \mathrm{pg} / \mathrm{mL}$ (NIBSC, Hertfordshire, UK). Aliquots of plasma from the QFT-IT tubes were stored at $-80^{\circ} \mathrm{C}$.

\section{IP-10 Assay}

The IP-10 concentrations were measured from the same supernatants of QFT-IT using a Human IP-10 ELISA Construction Kit (RayBiotech, Georgia, USA) according to the manufacturer's instructions. Samples were diluted 1:2 and tested in duplicates. The results were expressed in $\mathrm{pg} / \mathrm{mL}$ and classified as positive or negative according to a receiver operating curve (ROC). The test was considered indeterminate if the response of mitogen was less than 200 $\mathrm{pg} / \mathrm{mL}$ based on the earlier observation.(15)

\section{Clinical Diagnoses}

Based on the results of investigations, the children were diagnosed by a study doctor as: a) TB disease, either bacteriological confirmed or diagnosed clinically, if the child had positive result of sputum smear or Xpert MTB/ RIF or a child with well-defined symptoms of TB plus at least two of following three criteria: 1) positive IGRA result 2) radiological findings consistent with $\mathrm{TB}$, or 3 ) established history of close contact with a TB index case; b) latent TB infection (LTBI) if the child had a positive IGRA but did not meet the definition for TB disease; $c$ ) TB-exposed only if the child had a history of close contact with a TB index case, but did not meet the definitions for TB disease or LTBI; and d) non-TB if the child did not meet the criteria for TB disease, LTBI and TB-exposed only.

The study was approved by the Medical and Health Research Ethics Committee, Faculty of Medicine, Public Health and Nursing, Universitas Gadjah Mada, Yogyakarta, Indonesia (No. KE/FK/0450/EC/2017).

\section{Statistical Analysis}

Subjects with indeterminate IGRA results were excluded from analysis. Since the IFN- $\gamma$ and IP-10 values were not normally distributed, significant differences between the groups were determined using the nonparametric KruskalWallis test and the Mann-Whitney U-test for post-hoc analysis. The $p$-values were two-sided and considered significant if $<0.05$. Statistical analysis were performed using GraphPad Prism computer program (GraphPad Software Inc., San Diego, USA) and SPSS version 23.0 (IBM Corporation, New York, USA). The diagnostic performances of IP-10 were evaluated with a ROC curve analysis to determine the area under the curve (AUC), their $95 \%$ confidence intervals (CI), and the optimal cutoff levels. Tests concordance was assessed by k-statistics and Spearman Rank Correlation was used to correlate continuous variables.

\section{Results}

A total of 79 children were included in the study. The demographic and clinical characteristics of the children are shown in Table 1. Twelve children had TB disease while 16 were with LTBI, 40 were TB-exposed only, and 11 were non-TB. The median age of the children was 82 months (interquartile range (IQR): $38-141$ ), with 58\% of the children aged more than 5 years. The majority of the children had close contact with a TB patient.

The most common reported symptoms were cough (26.6\%), failure to thrive or weight loss $(22.8 \%)$, fever $(13.9 \%)$, and lethargy (10.1\%). Forty-three percent of children had abnormal CXR consistent with TB, with the commonest abnormality was hilar lymphadenopathy $(70.6 \%)$. Pleural effusion was identified in three

Table 1. Characteristics of the subjects.

\begin{tabular}{lc}
\hline \multicolumn{1}{c}{ Characteristics } & n (\%) \\
\hline Sex, male & $42(53.2)$ \\
\hline Age, years old & $33(41.8)$ \\
$0-4$ & $46(58.2)$ \\
$\geq 5$ & $67(84.8)$ \\
\hline Close contact to adult TB & $57(85.1)$ \\
Asymptomatic & $10(14.9)$ \\
\hline Symptomatic & $61(77.2)$ \\
\hline Nutritional status & $18(22.8)$ \\
Well-nourished & 0 \\
Moderate wasting & $79(100)$ \\
\hline Severe wasting & $64(81)$ \\
\hline BCG vaccination
\end{tabular}

TB: tuberculosis; BCG: bacille calmette guerin. 
symptomatic children and miliary pattern was in one symptomatic child. From 12 children with TB disease, three children were bacteriological confirmed, two of them had AFB sputum smear positive 1 and one of them had positive Xpert MTB/RIF. Positive result of IGRA was documented in $26(32.9 \%)$ children, negative result was in $44(55.7 \%)$, and indeterminate was in $9(11.4 \%)$.

\section{Plasma Level of IP-10}

Table 2 shows concentrations of IP-10 Nil, IP-10 Ag, IP10 Mit, IP-10 Ag-Nil, and IP-10 Mit-Nil in each group of children with TB disease, LTBI, TB-exposed only and nonTB. Figure 1 shows that children with TB infection, either with TB disease or LTBI, had significantly higher levels of IP-10 Ag-Nil compared to non-infected TB children, both TB-exposed only and non-TB. The level of antigenstimulated IP-10 was increased in active TB and in latent $\mathrm{TB}$, but there was no significant difference of stimulated levels of IP-10 between active TB and latent TB $(p=0.268)$. There was also no significant difference of IP-10 levels in response to unstimulated and mitogen between the four groups ( $p>0.05$ for all).

\section{Performance of IP-10 for the Diagnosis TB Infection and TB Disease}

The diagnostic value of IP-10 in identifying TB infection was evaluated by comparing the IP-10 levels between children with TB infection (either with TB disease or LTBI) and those who were TB-exposed only. The AUC of IP-10 AgNil in diagnosing TB infection was 0.970 (CI 95\%: 0.921$1.000, p=0.000)$. The cut-off IP-10 level to differentiate infected-TB child from healthy control was $408.74 \mathrm{pg} /$ $\mathrm{mL}$ (sensitivity: $92.3 \%$, specificity: $91.9 \%$ ). However, the diagnostic values of IP-10 in the unstimulated sample and in response to mitogen for diagnosis TB infection were poor $(p>0.05)$. The AUC of IP-10 Nil, IP-10 Ag-Nil, and IP-10 Mit-Nil for diagnosis of TB infection in children are shown in Figure 2.
To evaluate the diagnostic value of IP-10 in differentiating TB disease from TB infection, we compared the IP-10 levels of children with TB disease and those with LTBI. It was shown that it had poor diagnostic value with the AUC of IP-10 Nil was 0.528 (CI 95\%: 0.281-0.775, $p=0.813$ ), the AUC of IP-10 Ag-Nil was 0.631 (CI 95\%: $0.378-0.885, p=0.268)$, and the AUC of IP-10 Mit-Nil was 0.500 (CI 95\%: 0.251-0.749, $p=1.000$ ).

\section{Comparison and Concordance between the IP-10 Assay and QFT-IT Test}

We found that IP-10 was produced in higher level in plasma compared to IFN- $\gamma$ after stimulation of $M$. tuberculosis specific antigen (Figure 3). The overall indeterminate rate of IP-10 was lower compared to QFT-IT (7.6\% vs. 11.4\%). Moreover, we evaluated the correlation between the level of IP-10 and IFN- $\gamma$ in response to antigen stimulation and the result showed a significant and high correlation between the two markers ( $\mathrm{rs}=0.791, p=0.000$ ). Based on the IP-10 Ag-Nil cut-off previously identified for the diagnosis of TB infection, we scored the result positive and negative and evaluated the agreement between the IP-10 and QFTIT. Stratifying subjects according to their diagnosis, the concordance among all samples evaluated was optimal $(\mathrm{k}=0.837)$; in particular 24 patients scored positive in both assays, 34 patients scored negative, 3 patients scored positive in the IP-10 assay but negative in the QFT-IT test, and 2 patients scored negative in the IP-10 assay but positive in the QFT-IT test.

\section{Discussion}

Our study found increased IP-10 expression in $M$. tuberculosis-infected individuals. This finding are similar with other reports showing elevated IP-10 levels not only in adults $(4,10)$, but also in children $(9,11-16)$, in households or in close contact with active TB patients

Table 2. The IP-10 levels $(\mathrm{pg} / \mathrm{mL})$ in response to unstimulated, TB antigens, or mitogen.

\begin{tabular}{lccccc}
\hline & $\begin{array}{c}\text { TB-exposed Only } \\
(\mathbf{n = 3 7 )}\end{array}$ & $\begin{array}{c}\text { LTBI } \\
(\mathbf{n = 1 6})\end{array}$ & $\begin{array}{c}\text { TB Disease } \\
(\mathbf{n = 1 0})\end{array}$ & $\begin{array}{c}\text { Non-TB } \\
(\mathbf{n}=7)\end{array}$ & $\boldsymbol{p}$-value* \\
\hline IP-10 Nil & 259.74 & 321.97 & 371.10 & 516.46 & 0.338 \\
IP-10 Ag & 438.92 & 3705.87 & 4815.78 & 576.38 & $\leq 0.001$ \\
IP-10 Mit & 1938.96 & 2571.65 & 2308.76 & 2858.08 & 0.784 \\
IP-10 Ag-Nil & 73.62 & 3304.30 & 4364.44 & 59.92 & $\leq 0.001$ \\
IP-10 Mit-Nil & 1554.06 & 2227.81 & 2048.05 & 2204.48 & 0.816 \\
\hline
\end{tabular}

*tested with Kruskal-wallis. TB: tuberculosis; LTBI: latent TB infection; Nil: unstimulated; Ag: antigen; Mit: mitogen. 
$\mathbf{A}$

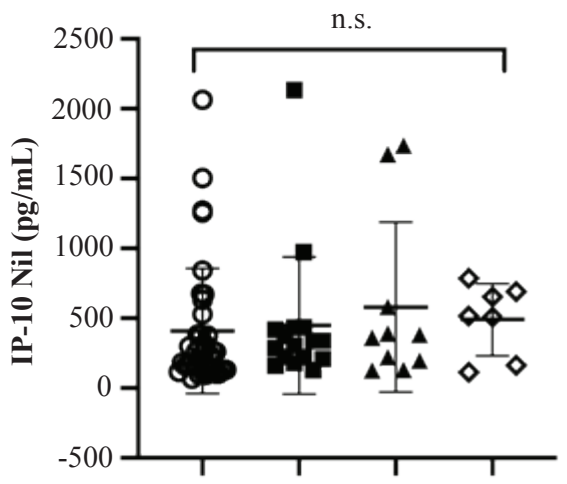

C

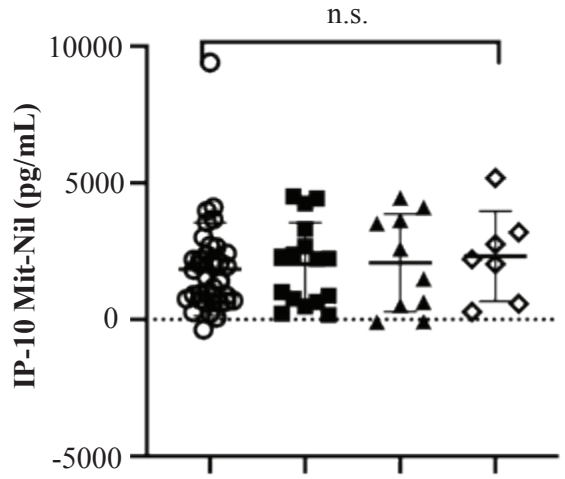

B

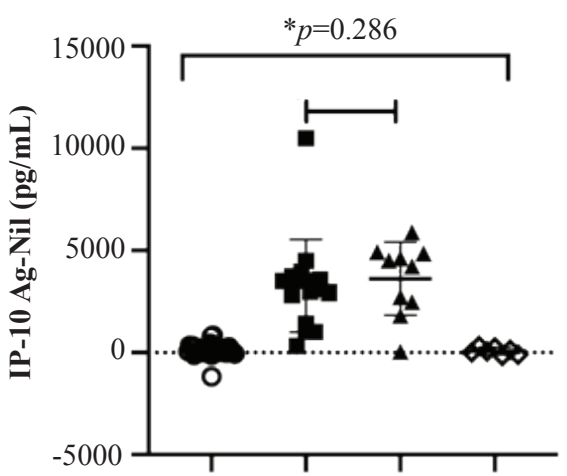

○ TB-exposed only

- LTBI

\ TB Disease

$\diamond$ Non-TB
Figure 1. The IP-10 levels levels among children with TB-exposed only, LTBI, TB disease, and non-TB. A: unstimulated (Nil) IP-10 levels; B: antigen-stimulated (Ag-Nil) IP-10 levels; C: mitogen-stimulated (Mit-Nil) IP-10 levels. Horizontal lines indicate median values, $\quad{ }^{*} p<0.05 . \quad$ IP-10: $\quad$ IFN- $\gamma$-nducible protein 10; TB: tuberculosis; LTBI: latent TB infection; ns: not significant.
$(17,18)$, in individuals with immunocompromised conditions (19), and in animals.(22) These findings confirmed the role of chemokine IP-10 as a key regulator of immune cell recruitment, in particular Th1 cells, towards the site of infection or inflammation which is important in TB pathogenesis, especially in granuloma formation.(23)

We also found that the diagnostic accuracy of IP-10 in diagnosing TB infection was good at the cut-off point of $408.74 \mathrm{pg} / \mathrm{mL}$ with sensitivity $92.3 \%$ and specificity $91.9 \%$, which is comparable with QFT-IT. Several other studies found similar result, which were recently summarized in a review by another article.(24) Performance of IP-10 has been reported as comparable to that of IFN- $\gamma$, and was better in children and the immunocompromised patients. $(11,14,25,26)$ Two other systematic reviews also proposed IP-10 as a powerful reference marker in TB infection, with moderate accuracy (overall pooled sensitivity of $73 \%$ and specificity of $83 \%$ ) for the detection of TB infection while performed alone. The diagnostic values were better when combined with other conventional tests such as TST or IGRA and clinical findings. $(27,28)$

The use of IP-10 in distinguishing latent and active TB is still debatable.(29) Although we found that stimulated IP-10 levels were higher in the active TB group compared to the latent TB group, this result was not statistically significant between the groups. Therefore, IP-10 could not be used to distinguish the two states of TB infection. Our findings support the previous studies demonstrating inability of IP-10 in identifying TB disease from latent TB infection. $(4,26,30)$ Contrary to our study, there were 3 studies in adults that showed the discriminative ability of IP-10 in diagnosing TB disease. One study indicated that IP-10, particularly in unstimulated plasma, serves as a potential biomarker to distinguish between active and latent TB with AUC of 0.86 , sensitivity $80 \%$, and specificity $80 \%$. (31) Another study also mentioned IP-10 Ag-Nil as a potent discriminative marker for active TB with AUC of 0.719, sensitivity $71.8 \%$, and specificity $71.1 \%$.(32) Discrimination between active and latent TB based on IP-10 assay was also reported by another research. They found IP-10 Ag-Nil with AUC of 0.8848 , sensitivity $69.7 \%$, and specificity $100 \%$ could distinguish active and latent TB. They also revealed that ratio IP-10 Ag-Nil/ IP-10 Mit-Nil with AUC of 0.9242, sensitivity $93.94 \%$ and specificity $90 \%$ was the strongest promising indicator for active disease vs latent TB.(33)

IP-10 plasma levels are often elevated during acute or chronic illness in inflammatory, infectious, and autoimmune diseases, as well as in several cancers.(34) Therefore, it is 


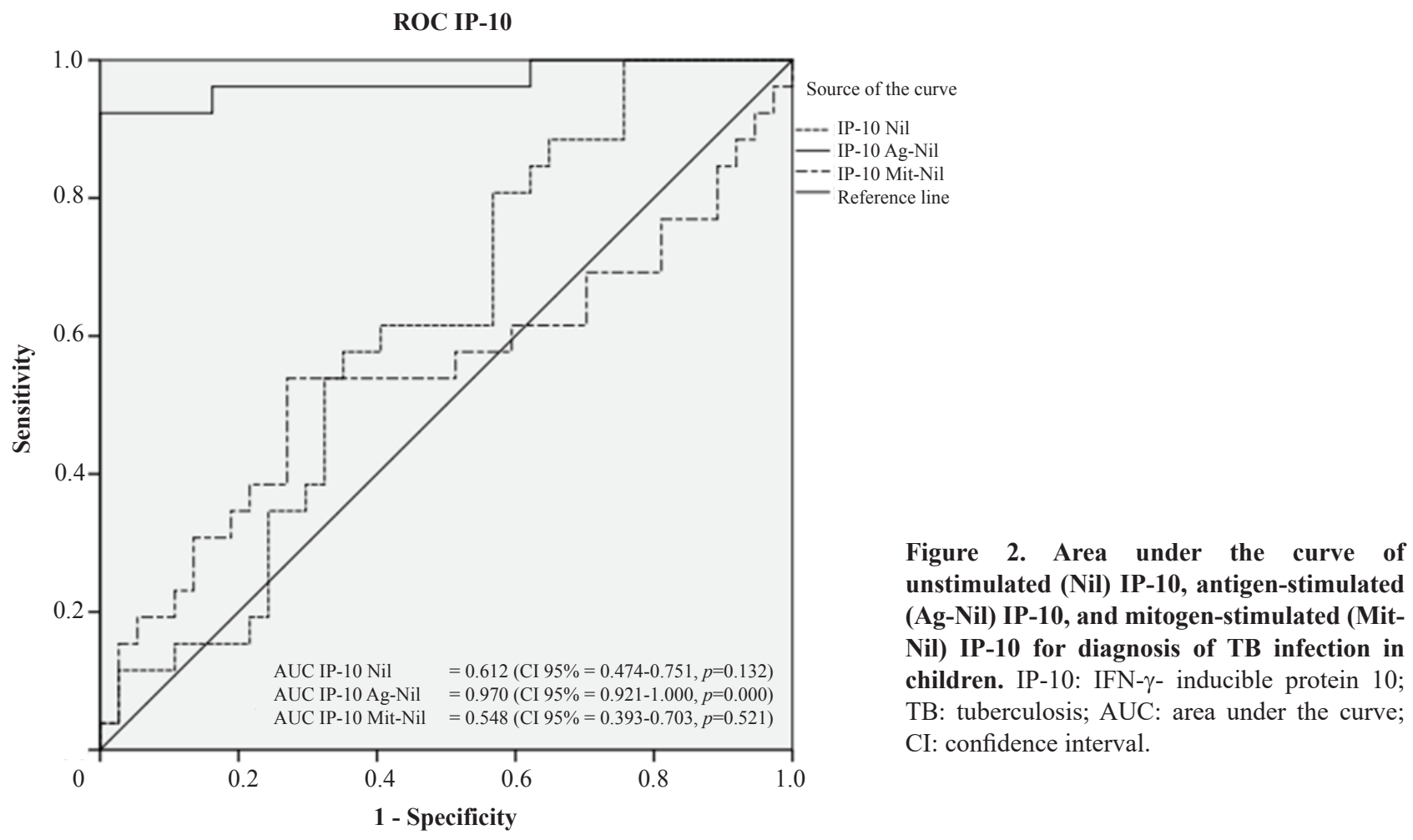

not surprising that we found higher $M$. tuberculosis-antigen stimulated IP-10 responses in children with active TB than in the latent TB group or the TB exposed only as the high risk control group. Another possible reason for this finding could be that IP-10 secretion may be highest during primary $M$. tuberculosis infection, as it appears to play a central role in orchestrating the recruitment of Th1 cells during granuloma formation into the M. tuberculosis-infected lung, which contributes to $M$. tuberculosis containment.(14) Contrary with our results, previous studies found the background level of IP-10 was much higher in TB infection than TB

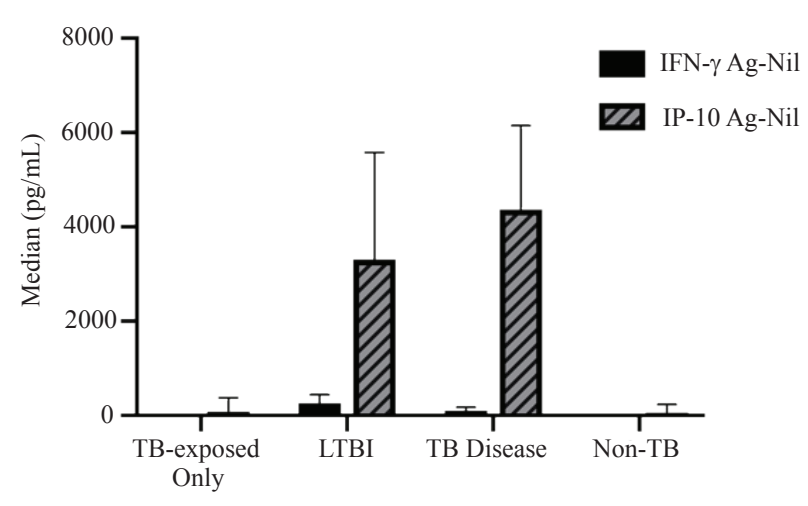

Figure 3. The comparison between median level of antigenstimulated IP-10 and IFN- $\gamma$ among children with TB-exposed only, LTBI, TB disease, and non-TB. IP-10: IFN- $\gamma$-nducible protein 10; TB: tuberculosis; LTBI: latent TB infection. disease, and they speculated that this could be because of a chronic state of inflammation due to the immune response in attempting to control the TB infection.(26) However, active TB has an immunosuppressive effect which changes cytokine responses providing an alternative explanation. A recent study raised a new topic on IP-10 antagonism that might be able to bridge the contrary findings. In addition to high levels of IP-10 in active TB patients, they also found high levels of IP-10 antagonism, which can inactivate IP10 then reducing its chemotactic functions in directing migration of Th1 cells to the site of infection.(35) How the chemokines, especially IP-10, drive the balance between protective and damaging inflammation, as well as the levels of inflammation required for $M$. tuberculosis containment remains a question that needs further investigation.

Several studies that have been conducted in children have not shown good agreement on the diagnostic performance of IP-10 in M. tuberculosis infection with a wide range of sensitivity (63-95\%) and specificity (53-100\%). These results can be caused by different endemic level of TB and wide variation in the use of reference standards for the confirmation of cases, types of samples, dilution of samples, choice of M. tuberculosis antigen for stimulation, kits, test methods, and thresholds.(6,12,36-38) Modification of several components was suggested by another publication in an effort to increase IP-10 accuracy to distinguish active and latent conditions in TB. These include using 
M. tuberculosis antigen stimulant specifically expressed under replicative or dormant conditions, taking samples from site of infection or using combination of samples e.g., blood and urine, extending the duration of incubation, and combining IP-10 with other biomarkers.(39)

The strength of this study is that we recruited symptomatic and asymptomatic children with active and passive approach of case finding, which allowed us to have wide spectrum of condition, from TB-exposed only, TB infection to TB disease. We also recruited children from both inpatient and outpatient clinics, therefore the spectrum of the disease was wide. Although IP-10 has not been able to replace the role of IFN- $\gamma$ in detection of TB disease, IP-10 has technical advantages for the diagnosis of TB in children. This study and several previous studies found the rates of indeterminate results of IP-10 in children seemed to show a tendency towards fewer counts compared to IFN- $\gamma$. (7) Expression of IP-10 is robust in infected TB children and quantitatively higher $>100 \mathrm{x}$ than IFN- $\gamma$.(22) It can be caused of IP-10 is a downstream marker compared to IFN- $\gamma$ and other classical T cell cytokines in cell mediated immune respon assays.(24) High concentrations of IP10 allow detection of small specimens such as taking from blood capillary from the fingertips so that it is more feasible conducted in children.(7) The stable nature of IP10 in the dried plasma spots in the filter paper at ambient temperature ease in transportation while also allowing for the development of rapid tests in the form of a lateral flow test such as HIV testing and pregnancy test.(40)

However, several limitations should be considered. First, the lack of a gold standard to diagnose TB in child may cause a source population bias. We classified patients based on combination of clinical features, microbiology confirmation, evidence of infection include immunological evidence and contact history, and CXR finding. Following up children to evaluate the response to TB treatment might increase the accuracy of TB disease. Second, the small sample population could produce type II error in the statistical analysis.

\section{Conclusion}

IP-10 provides a new specific biomarker for TB infection, but IP-10 does not appear to be superior than IFN $\gamma$ in distinguishing active TB disease from latent TB infection. These findings contribute to our understanding of IP-10 as a potential inflammatory marker in the blood. Studies with larger populations are needed to confirm these finding involving more standardized IP-10 assay techniques and validated cutoffs.

\section{Acknowledgements}

The authors gratefully extend their thanks to the Directorate of Research and Community Service from the Ministry of Research, Technology and Higher Education Republic of Indonesia for funding this research through the "Doctoral Dissertation Research Scheme (No. 102-16/UN7.P4.3/ PP/2018)".

\section{References}

1. Oliwa JN, Karumbi JM, Marais BJ, Madhi SA, Graham SM. Tuberculosis as a cause or comorbidity of childhood pneumonia in tuberculosis-endemic areas: a systematic review. Lancet Respir Med. 2015; 3: 235-43.

2. World Health Organization (WHO). Global tuberculosis report 2017. Geneva: WHO; 2017.

3. Dunn JJ, Starke JR, Revell PA. Laboratory diagnosis of Mycobacterium tuberculosis infection and disease in children. J Clin Microbiol. 2016; 54: 1434-41.

4. La Manna MP, Orlando V, Li Donni P, Sireci G, Di Carlo P, Cascio A, et al. Identification of plasma biomarkers for discrimination between tuberculosis infection/disease and pulmonary non tuberculosis disease. PLOS ONE. 2018; 13: e0192664. doi: 10.1371/journal. pone. 0192664 .

5. Holm LL, Rose MV, Kimaro G, Bygbjerg IC, Mfinanga SG, Ravn P, et al. A comparison of Interferon- $\gamma$ and IP-10 for the diagnosis of tuberculosis. Pediatrics. 2014; 134: e1568-75.

6. Chegou NN, Detjen AK, Thiart L, Walters E, Mandalakas AM, Hesseling AC, et al. Utility of host markers detected in quantiferon supernatants for the diagnosis of tuberculosis in children in a highburden setting. PLoS ONE. 2013; 8: e64226. doi: 10.1371/journal. pone. 0064226 .

7. Ruhwald M, Aabye MG, Ravn P. IP-10 release assays in the diagnosis of tuberculosis infection: current status and future directions. Expert Rev Mol Diagn. 2012; 12: 175-87.

8. Aabye MG, Latorre I, Diaz J, Maldonado J, Mialdea I, Eugen-Olsen J. Dried plasma spots in the diagnosis of tuberculosis: IP-10 release assay on filter paper. Eur Respir J. 2013; 42: 495-503.

9. Jenum S, Dhanasekaran S, Ritz C, Macaden R, Doherty TM, Grewal HMS. Added value of IP-10 as a read-out of Mycobacterium tuberculosis: Specific immunity in young children. Pediatr Infect Dis J. 2016; 35: 1336-8.

10. Wang S, Li Y, Shen Y, Wu J, Gao Y, Zhang S, et al. Screening and identification of a six-cytokine biosignature for detecting TB infection and discriminating active from latent TB. J Transl Med 2018; 16: 2016. doi: 10.1186/s12967-018-1572-x.

11. Latorre I, Díaz J, Mialdea I, Serra-Vidal M, Altet N, Prat C, et al. IP-10 is an accurate biomarker for the diagnosis of tuberculosis in children. J Infect. 2014; 69: 590-9.

12. Armand M, Chhor V, de Lauzanne A, Guérin-El Khourouj V, Pédron $\mathrm{B}$, Jeljeli M, et al. Cytokine responses to quantiferon peptides in 
pediatric tuberculosis: A pilot study. J Infect . 2014; 68: 62-70.

13. Alsleben N, Ruhwald M, Rüssmann H, Marx FM, Wahn U, Magdorf $\mathrm{K}$. Interferon-gamma inducible protein 10 as a biomarker for active tuberculosis and latent tuberculosis infection in children: A casecontrol study. Scand J Infect Dis. 2012; 44: 256-62.

14. Lighter J, Rigaud M, Huie M, Peng CH, Pollack H. Chemokine IP-10: an adjunct marker for latent tuberculosis infection in children. Int $\mathrm{J}$ Tuberc Lung Dis. 2009; 13: 731-6.

15. Ruhwald M, Petersen J, Kofoed K, Nakaoka H, Cuevas LE, Lawson $\mathrm{L}$, et al. Improving T-Cell assays for the diagnosis of latent TB infection: Potential of a diagnostic test based on IP-10. PLoS ONE. 2008; 3(8): e2858. doi: 10.1371/journal.pone.0002858.

16. Petrucci R, Amer NA, Gurgel RQ, Sherchand JB, Doria L, Lama C, et al. Interferon gamma, interferon-gamma-induced-protein 10, and tuberculin responses of children at high risk of tuberculosis infection. Pediatr Infect Dis J. 2008; 27: 1073-7.

17. Biraro IA, Kimuda S, Egesa M, Cose S, Webb EL, Joloba M, et al. The use of interferon gamma inducible protein 10 as a potential biomarker in the diagnosis of latent tuberculosis infection in Uganda. PLOS ONE. 2016; 11: e0146098. doi: 10.1371/journal. pone.0146098.

18. Syed Ahamed Kabeer B, Paramasivam P, Raja A. Interferon gamma and interferon gamma inducible protein-10 in detecting tuberculosis infection. J Infect. 2012; 64: 573-9.

19. Goletti D, Raja A, Syed Ahamed Kabeer B, Rodrigues C, Sodha A, Carrara S, et al. Is IP-10 an accurate marker for detecting M. tuberculosis-specific response in HIV-infected persons? PLoS ONE. 2010: 5(9): e12577. doi: 10.1371/journal.pone.0012577.

20. Marais BJ. Well defined symptoms are of value in the diagnosis of childhood pulmonary tuberculosis. Arch Dis Child. 2005; 90: 1162-5.

21. World Health Organization (WHO). WHO child growth standards: length/height-for-age, weight-for-age, weight-for-length, weightfor-height and body mass index-for-age; methods and development. Geneva: WHO; 2006.

22. Aabye MG, Eugen-Olsen J, Werlinrud AM, Holm LL, Tuuminen T, Ravn $\mathrm{P}$, et al. A simple method to quantitate IP-10 in dried blood and plasma spots. PLoS ONE. 2012; 7: e39228. doi: 10.1371/ journal.pone.0039228.

23. Slight SR, Khader SA. Chemokines shape the immune responses to tuberculosis. Cytokine Growth Factor Rev. 2013; 24: 105-13.

24. Chegou NN, Heyckendorf J, Walzl G, Lange C, Ruhwald M. Beyond the IFN-g horizon: biomarkers for immunodiagnosis of infection with Mycobacterium tuberculosis. Eur Respir J. 2014; 43: 1472-86.

25. Syed Ahamed Kabeer B, Sikhamani R, Raja A. Comparison of interferon gamma-inducible protein-10 and interferon gammabased QuantiFERON TB Gold assays with tuberculin skin test in HIV-infected subjects. Diagn Microbiol Infect Dis. 2011; 71: 23643.

26. Whittaker E, Gordon A, Kampmann B. Is IP-10 a better biomarker for active and latent tuberculosis in children than IFN $\gamma$ ? PLoS ONE. 2008; 3: e3901. doi: 10.1371/journal.pone.0003901.
27. Guo SJ, Jia LQ, Hu QJ, Long HY, Pang CS, Wen FQ. Diagnostic accuracy of interferon gamma-induced protein 10 for tuberculosis: a meta-analysis. Int J Clin Exp Med. 2014; 7(1): 93-100.

28. Firoozeh A, Khaledi A, Rezaee SA, Ghazvini K. Diagnostic accuracy of IP-10 biomarker for Mycobacterium tuberculosis: A systematic review. Der Pharmacia Lettre. 2016; 8: 294-8.

29. Venturini E, Remaschi G, Berti E, Montagnani C, Galli L, de Martino $\mathrm{M}$, et al. What steps do we need to take to improve diagnosis of tuberculosis in children? Expert Rev Anti Infect Ther. 2015; 13: 907-22.

30. Frahm M, Goswami ND, Owzar K, Hecker E, Mosher A, Cadogan $\mathrm{E}$, et al. Discriminating between latent and active tuberculosis with multiple biomarker responses. Tuberculosis. 2011; 91: 250-6.

31. Yao X, Liu Y, Liu Y, Liu W, Ye Z, Zheng C, et al. Multiplex analysis of plasma cytokines/chemokines showing different immune responses in active TB patients, latent TB infection and healthy participants. Tuberculosis. 2017; 107: 88-94.

32. You E, Kim MH, Lee WI, Kang SY. Evaluation of IL-2, IL-10, IL-17 and IP-10 as potent discriminative markers for active tuberculosis among pulmonary tuberculosis suspects. Tuberculosis. 2016; 99: $100-8$.

33. Jeong YH, Hur YG, Lee H, Kim S, Cho JE, Chang J, et al. Discrimination between active and latent tuberculosis based on ratio of antigen-specific to mitogen-induced IP-10 production. J Clin Microbiol. 2015; 53: 504-10.

34. Liu M, Guo S, Hibbert JM, Jain V, Singh N, Wilson NO, et al. CXCL10/IP-10 in infectious diseases pathogenesis and potential therapeutic implications. Cytokine Growth Factor Rev. 2011; 22: 121-30.

35. Blauenfeldt T, Petrone L, del Nonno F, Baiocchini A, Falasca L, Chiacchio T, et al. Interplay of DDP4 and IP-10 as a Potential Mechanism for Cell Recruitment to Tuberculosis Lesions. Front Immunol. 2018; 9: 1456. doi: 10.3389/fimmu.2018.01456.

36. Amanatidou V, Critselis E, Trochoutsou A, Soldatou A, Benetatou $\mathrm{K}$, Spyridis $\mathrm{N}$, et al. Interferon gamma inducible protein-10 in the diagnosis of paediatric tuberculosis infection in a low TB incidence country. Int J Tuberc Lung Dis. 2015; 19: 1463-9.

37. Petrone L, Cannas A, Aloi F, Nsubuga M, Serumkuma J, Nazziwa RA, et al. Blood or urine IP-10 cannot discriminate between active tuberculosis and respiratory diseases different from tuberculosis in children. BioMed Res Int. 2015; 2015: 589471. doi: 10.1155/2015/589471.

38. Tebruegge M, Dutta B, Donath S, Ritz N, Forbes B, CamachoBadilla $\mathrm{K}$, et al. Mycobacteria-specific cytokine responses detect tuberculosis infection and distinguish latent from active tuberculosis. Am J Respir Crit Care Med. 2015; 192: 485-99.

39. Clifford V. Improving diagnostic tests for tuberculosis: mycobacterial antigen-stimulated cytokine biomarkers for diagnosis and monitoring therapy [Dissertation]. Melbourne: The University of Melbourne; 2017.

40. Tuuminen T, Salo E, Kotilainen H, Ruhwald M. Evaluation of the filter paper IP-10 tests in school children after exposure to tuberculosis: a prospective cohort study with a 4-year follow-up. BMJ Open. 2012; 2: e001751. doi: 10.1136/bmjopen-2012-001751. 